\title{
ON NORMAL FORMS OF SINGULAR LEVI-FLAT REAL ANALYTIC HYPERSURFACES
}

\author{
ARTURO FERNÁNDEZ-PÉREZ
}

\begin{abstract}
Let $F(z)=\mathcal{R e}(P(z))+$ h.o.t be such that $M=(F=0)$ defines a germ of real analytic Levi-flat at $0 \in \mathbb{C}^{n}, n \geq 2$, where $P(z)$ is a homogeneous polynomial of degree $k$ with an isolated singularity at $0 \in \mathbb{C}^{n}$ and Milnor number $\mu$. We prove that there exists a holomorphic change of coordinate $\phi$ such that $\phi(M)=(\mathcal{R} e(h)=0)$, where $h(z)$ is a polynomial of degree $\mu+1$ and $j_{0}^{k}(h)=P$.
\end{abstract}

\section{Introduction and Statement of the Results}

Let $M$ be a germ at $0 \in \mathbb{C}^{n}$ of a real codimension one irreducible analytic set. For the sake of simplicity we will denote germs and representative of germs by the same letter. Since $M$ is real analytic of codimension one and irreducible, it can be defined in $\mathbb{C}^{n}$ by $(F=0)$, where $F$ is an irreducible germ of real analytic function. The singular set of $M$ is defined by $\operatorname{sing}(M)=(F=0) \cap(d F=0)$ and its smooth part $(F=0) \backslash(d F=0)$ will denoted by $M^{*}$. The Levi distribution $L$ on $M^{*}$ is defined by $L_{p}:=\operatorname{ker}(\partial F(p)) \subset T_{p} M^{*}=\operatorname{ker}(d F(p))$, for any $p \in M^{*}$.

Definition 1.1. We say $M$ is Levi-flat if the Levi distribution on $M^{*}$ is integrable.

Remark 1.2. The integrability condition of $L$ implies that $M^{*}$ is tangent to a real codimension one foliation $\mathcal{L}$. Since the hyperplanes $L_{p}, p \in M^{*}$, are complex, the leaves of $\mathcal{L}$ are complex codimension one holomorphic submanifolds immersed on $M^{*}$.

Remark 1.3. If the hypersurface $M$ is defined by $(F=0)$ then the Levi distribution $L$ on $M$ can be defined by the real analytic 1-form $\eta=i(\partial F-$ $\bar{\partial} F)$, which will be called the Levi 1-form of $F$. The integrability condition is equivalent to $\left.(\partial F-\bar{\partial} F) \wedge \partial \bar{\partial} F\right|_{M^{*}}=0$

In the case of a real analytic smooth Levi-flat hypersurface $M$ in $\mathbb{C}^{n}$, its local structure is very well understood, according to E. Cartan, around each $p \in M$ we can find local holomorphic coordinates $z_{1}, \ldots, z_{n}$ such that $M=\left\{\mathcal{R} e\left(z_{1}\right)=0\right\}$.

More recently D. Burns and X. Gong [B-G] have proved an analogous result in the case $M=F^{-1}(0)$ Levi-flat, where $F:\left(\mathbb{C}^{n}, 0\right) \rightarrow(\mathbb{R}, 0), n \geq 2$, is a germ of real analytic function such that

$$
F\left(z_{1}, \ldots, z_{n}\right)=\mathcal{R} e\left(z_{1}^{2}+\ldots+z_{n}^{2}\right)+\text { h.o.t. }
$$

2010 Mathematics Subject Classification. Primary 32V40 - 37F75.

Key words and phrases. Levi-flat hypersurfaces - Holomorphic foliations. 
They show that there exists a germ of biholomorphism $\phi:\left(\mathbb{C}^{n}, 0\right) \rightarrow$ $\left(\mathbb{C}^{n}, 0\right)$ such that $\phi(M)=\left(\mathcal{R} e\left(z_{1}^{2}+\ldots+z_{n}^{2}\right)=0\right)$.

In [C-L], the authors prove the above result by using the theory of holomorphic foliations. In this paper we are interested in finding similar normal forms in a situation more general. Our main result is the following:

Theorem 1. Let $M=F^{-1}(0)$, where $F:\left(\mathbb{C}^{n}, 0\right) \rightarrow(\mathbb{R}, 0), n \geq 2$, be a germ of irreducible real analytic function such that

(1) $F\left(z_{1}, \ldots, z_{n}\right)=\operatorname{Re} e\left(P\left(z_{1}, \ldots, z_{n}\right)\right)+$ h.o.t, where $P$ is a homogeneous polynomial of degree $k$ with an isolated singularity at $0 \in \mathbb{C}^{n}$.

(2) The Milnor number of $P$ at $0 \in \mathbb{C}^{n}$ is $\mu$.

(3) $M$ is Levi-flat.

Then there exists a germ of biholomorphism $\phi:\left(\mathbb{C}^{n}, 0\right) \rightarrow\left(\mathbb{C}^{n}, 0\right)$ such that $\phi(M)=(\mathcal{R e}(h)=0)$, where $h(z)$ is a polynomial of degree $\mu+1$ and $j_{0}^{k}(h)=P$.

Remark 1.4. In particular, we obtain the result of $[B-G]$.

Theorem 2. In the same spirit we have the following generalization: Let $M=F^{-1}(0)$, where $F:\left(\mathbb{C}^{n}, 0\right) \rightarrow(\mathbb{R}, 0), n \geq 3$, be a germ of irreducible real analytic function such that

(1) $F\left(z_{1}, \ldots, z_{n}\right)=\mathcal{R} e\left(Q\left(z_{1}, \ldots, z_{n}\right)\right)+$ h.o.t, where $Q$ is a quasihomogeneous polynomial of degree $d$ with an isolated singularity at $0 \in \mathbb{C}^{n}$.

(2) $M$ is Levi-flat.

Then there exists a germ of biholomorphism $\phi:\left(\mathbb{C}^{n}, 0\right) \rightarrow\left(\mathbb{C}^{n}, 0\right)$ such that

$$
\phi(M)=\left(\mathcal{R} e\left(Q(z)+\sum_{j} c_{j} e_{j}(z)\right)=0\right),
$$

where $e_{1}, \ldots, e_{s}$ are the elements of the monomial basis of the local algebra of $Q$ such that $\operatorname{deg}\left(e_{j}\right)>d$ and $c_{j} \in \mathbb{C}$.

\section{Acknowledgments}

I want to express my thanks to my advisor Alcides Lins Neto, for valuable conversations. The author would like to thank also IMPA, where this work was developed. I also want to thank the referee for his suggestions.

\section{Preliminaries}

Let us fix some notations that will be used from now on.

(1) $\mathcal{O}_{n}$ : The ring of germs of holomorphic functions at $0 \in \mathbb{C}^{n}$. $\mathcal{O}(U)=$ set of holomorphic functions in the open set $U \subset \mathbb{C}^{n}$.

(2) $\mathcal{O}_{n}^{*}=\left\{f \in \mathcal{O}_{n} / f(0) \neq 0\right\}$. $\mathcal{O}^{*}(U)=\{f \in \mathcal{O}(U) / f(z) \neq 0, \forall z \in U\}$.

(3) $\mathcal{M}_{n}=\left\{f \in \mathcal{O}_{n} / f(0)=0\right\}$ maximal ideal of $\mathcal{O}_{n}$.

(4) $\mathcal{A}_{n}$ : The ring of germs at $0 \in \mathbb{C}^{n}$ of complex valued real analytic functions.

(5) $\mathcal{A}_{n \mathbb{R}}$ : The ring of germs at $0 \in \mathbb{C}^{n}$ of real valued real analytic functions. Note that $F \in \mathcal{A}_{n}$ is in $\mathcal{A}_{n \mathbb{R}}$ if and only if $F=\bar{F}$.

(6) $\operatorname{Diff}\left(\mathbb{C}^{n}, 0\right)$ : The group of germs at $0 \in \mathbb{C}^{n}$ of holomorphic diffeomorphisms $f:\left(\mathbb{C}^{n}, 0\right) \rightarrow\left(\mathbb{C}^{n}, 0\right)$ with the operation of composition.

(7) $j_{0}^{k}(f):$ The $k$-jet at $0 \in \mathbb{C}^{n}$ of $f \in \mathcal{O}_{n}$. 
Definition 2.1. Two germs $f, g \in \mathcal{O}_{n}$ are said to be right equivalent, if there exists $\phi \in \operatorname{Diff}\left(\mathbb{C}^{n}, 0\right)$ such that $f \circ \phi^{-1}=g$.

The local algebra of $f \in \mathcal{O}_{n}$ is by definition

$$
A_{f}=\mathcal{O}_{n} /\left(\partial f / \partial z_{1}, \ldots, \partial f / \partial z_{n}\right) .
$$

Definition 2.2. Define by $\mu(f, 0):=\operatorname{dim} A_{f}$, the Milnor number of $f$ at $0 \in \mathbb{C}^{n}$.

Morse Lemma can now be rephrased by saying that if $0 \in \mathbb{C}^{n}$ is an isolated singularity of $f$ with Milnor number $\mu(f, 0)=1$ then $f$ is right equivalent to its second jet. The next lemma is a generalization of Morse's Lemma. We refer to [A-G-V], pg.121.

Lemma 2.3. Suppose $0 \in \mathbb{C}^{n}$ is an isolated singularity of $f \in \mathcal{M}_{n}$ with Milnor number $\mu$. Then $f$ is right equivalent to $j_{0}^{\mu+1}(f)$.

2.1. The complexification. In this section we state some general facts about complexification of germs of real analytic functions.

Given $F \in \mathcal{A}_{n}$; we can write its Taylor series at $0 \in \mathbb{C}^{n}$ as

$$
F(z)=\sum_{\mu, \nu} F_{\mu \nu} z^{\mu} \bar{z}^{\nu}
$$

where $F_{\mu \nu} \in \mathbb{C}, \mu=\left(\mu_{1}, \ldots, \mu_{n}\right), \nu=\left(\nu_{1}, \ldots, \nu_{n}\right), z^{\mu}=z_{1}^{\mu_{1}} \ldots z_{n}^{\mu_{n}}, \bar{z}^{\nu}=$ $\bar{z}_{1}^{\nu_{1}} \ldots \bar{z}_{n}^{\nu_{n}}$. When $F \in \mathcal{A}_{n \mathbb{R}}$ then the coefficients $F_{\mu \nu}$ satisfy

$$
\bar{F}_{\mu \nu}=F_{\nu \mu} .
$$

The complexification $F_{\mathbb{C}} \in \mathcal{O}_{2 n}$ of $F$ is defined by the series

$$
F_{\mathbb{C}}(z, w)=\sum_{\mu, \nu} F_{\mu \nu} z^{\mu} w^{\nu} .
$$

If $F \in \mathcal{A}_{n \mathbb{R}}, F(0)=0$ and $M=F^{-1}(0)$ defines a Levi-flat, the complexification $\eta_{\mathbb{C}}$ of its Levi 1-form $\eta=i(\partial F-\bar{\partial} F)$ can be written as

$$
\eta_{\mathbb{C}}=i\left(\partial_{z} F_{\mathbb{C}}-\partial_{w} F_{\mathbb{C}}\right)=i \sum_{\mu, \nu}\left(F_{\mu \nu} w^{\nu} d\left(z^{\mu}\right)-F_{\mu \nu} z^{\mu} d\left(w^{\nu}\right)\right) .
$$

The complexification $M_{\mathbb{C}}$ of $M$ is defined as $M_{\mathbb{C}}=F_{\mathbb{C}}^{-1}(0)$ and its smooth part is $M_{\mathbb{C}}^{*}=M_{\mathbb{C}} \backslash\left(d F_{\mathbb{C}}=0\right)$. The integrability condition of $\eta=i(\partial F-$ $\bar{\partial} F)\left.\right|_{M^{*}}$ implies that $\left.\eta_{\mathbb{C}}\right|_{M_{\mathbb{C}}^{*}}$ is integrable. Therefore $\left.\eta_{\mathbb{C}}\right|_{M_{\mathbb{C}}^{*}}=0$ defines a foliation $\mathcal{L}_{\mathbb{C}}$ on $M_{\mathbb{C}}^{*}$ that will be called the complexification of $\mathcal{L}$.

Definition 2.4. The algebraic dimension of $\operatorname{sing}(M)$ is the complex dimension of the singular set of $M_{\mathbb{C}}$.

Consider a germ at $0 \in \mathbb{C}^{2}$ of real analytic Levi-flat $M=(F=0)$, where $F$ is irreducible in $\mathcal{A}_{2 \mathbb{R}}$. Let $F_{\mathbb{C}}, M_{\mathbb{C}}=\left(F_{\mathbb{C}}=0\right) \subset\left(\mathbb{C}^{4}, 0\right)$ and $M_{\mathbb{C}}^{*}$ be as before. We will assume that the power series that defines $F_{\mathbb{C}}$ converges in a neighborhood of $\bar{\triangle}=\left\{(z, w) \in \mathbb{C}^{4} /|z|,|w| \leq 1\right\}$, so that $F_{\mathbb{C}}(z, \bar{z})=F(z)$ for all $|z| \leq 1$.

Let $V:=M_{\mathbb{C}}^{*} \backslash \operatorname{sing}\left(\left.\eta_{\mathbb{C}}\right|_{M_{\mathbb{C}}}\right)$ and denote $L_{p}$ the leaf of $\mathcal{L}_{\mathbb{C}}$ through $p$, where $p \in V$. In this situation we have the following important Lemma of [C-L]. 
Lemma 2.5. In the above situation, for any $p=\left(z_{0}, w_{0}\right) \in V$ the leaf $L_{p}$ is closed in $M_{\mathbb{C}}^{*}$.

In the proof of theorem 1 we will use the following result of [C-L].

Theorem 2.6. Let $M=F^{-1}(0)$ be a germ of an irreducible real analytic Levi-flat hypersurface at $0 \in \mathbb{C}^{n}, n \geq 2$, with Levi 1-form $\eta=i(\partial F-\bar{\partial} F)$. Assume that the algebraic dimension of $\operatorname{sing}(M) \leq 2 n-4$. Then there exists an unique germ at $0 \in \mathbb{C}^{n}$ of holomorphic codimension one foliation $\mathcal{F}_{M}$ tangent to $M$, if one of the following conditions is fulfilled:

(1) $n \geq 3$ and $\operatorname{cod}_{M_{\mathbb{C}}^{*}}\left(\operatorname{sing}\left(\left.\eta_{\mathbb{C}}\right|_{M_{\mathbb{C}}^{*}}\right)\right) \geq 3$.

(2) $n \geq 2, \operatorname{cod}_{M_{\mathbb{C}}^{*}}\left(\operatorname{sing}\left(\left.\eta_{\mathbb{C}}\right|_{M_{\mathbb{C}}^{*}}\right)\right) \geq 2$ and $\mathcal{L}_{\mathbb{C}}$ has a non-constant holomorphic first integral.

Moreover, in both cases the foliation $\mathcal{F}_{M}$ has a non-constant holomorphic first integral $f$ such that $M=(\operatorname{Re}(f)=0)$.

\section{Proof of theorem 1}

Let $M=F^{-1}(0) \subset\left(\mathbb{C}^{n}, 0\right)$ be a Levi-flat, where $F(z)=\mathcal{R} e(P(z))+$ h.o.t with $P$ be a homogeneous polynomial of degree $k \geq 2$ with an isolated singularity at $0 \in \mathbb{C}^{n}$ and Milnor number $\mu$. We want to prove that there exists $\phi \in \operatorname{Diff}\left(\mathbb{C}^{n}, 0\right)$ such that $\phi(M)=(\mathcal{R} e(h)=0)$, where $h$ is a polynomial of degree $\mu+1$.

The idea is to use theorem 2.6 to prove that there exists a germ $f \in \mathcal{O}_{n}$ such that the foliation $\mathcal{F}$ defined by $d f=0$ is tangent to $M$ and $M=$ $(\mathcal{R} e(f)=0)$. The foliation $\mathcal{F}$ can viewed as an extension to a neighborhood of $0 \in \mathbb{C}^{n}$ of the Levi foliation $\mathcal{L}$ on $M^{*}$.

Suppose for a moment that $M=(\mathcal{R} e(f)=0)$ and let us conclude the proof. Without lost of generality, we can suppose that $f$ is not a power in $\mathcal{O}_{n}$. In this case $\mathcal{R} e(f)$ is irreducible (cf. [C-L]). This implies that $\mathcal{R} e(f)=U . F$, where $U \in \mathcal{A}_{n \mathbb{R}}$ and $U(0) \neq 0$. Let $\sum_{j \geq k} f_{j}$ be the taylor series of $f$, where $f_{j}$ is a homogeneous polynomial of degree $j, j \geq k$. Then

$$
\mathcal{R} e\left(f_{k}\right)=j_{0}^{k}(\mathcal{R} e(f))=j_{0}^{k}(U . F)=U(0) \cdot \mathcal{R} e\left(P\left(z_{1}, \ldots, z_{n}\right)\right) .
$$

Hence $f_{k}\left(z_{1}, \ldots, z_{n}\right)=U(0) \cdot P\left(z_{1}, \ldots, z_{n}\right)$. We can suppose that $U(0)=1$, so that

$$
f(z)=P(z)+\text { h.o.t }
$$

In particular, $\mu=\mu(f, 0)=\mu(P, 0), f \in \mathcal{M}_{n}$, because $P$ has isolated singularity at $0 \in \mathbb{C}^{n}$. Hence by lemma 2.3, $f$ is right equivalent to $j_{0}^{\mu+1}(f)$, i.e. there exists $\phi \in \operatorname{Diff}\left(\mathbb{C}^{n}, 0\right)$ such that $h:=f \circ \phi^{-1}=j_{0}^{\mu+1}(f)$. Therefore, $\phi(M)=(\mathcal{R} e(h)=0)$ and this will conclude the proof of theorem 1.

Let us prove that we can apply theorem 2.6. We can write

$$
F(z)=\mathcal{R} e\left(P\left(z_{1}, \ldots, z_{n}\right)\right)+H\left(z_{1}, \ldots, z_{n}\right),
$$

where $H:\left(\mathbb{C}^{n}, 0\right) \rightarrow(\mathbb{R}, 0)$ is a germ of real-analytic function and $j_{0}^{k}(H)=0$. For simplicity, we assume that $P$ has real coefficients. Then we get the complexification

$$
F_{\mathbb{C}}(z, w)=\frac{1}{2}(P(z)+P(w))+H_{\mathbb{C}}(z, w)
$$


and $M_{\mathbb{C}}=F_{\mathbb{C}}^{-1}(0) \subset\left(\mathbb{C}^{2 n}, 0\right)$. In the general case, replacing $P(w)=\sum a_{j} w^{j}$ by $\tilde{P}(w)=\sum \bar{a}_{j} w^{j}$, we will recover each step of proof.

Since $P(z)$ has an isolated singularity at $0 \in \mathbb{C}^{n}$, we get $\operatorname{sing}\left(M_{\mathbb{C}}\right)=$ $\{0\}$, and so the algebraic dimension of $\operatorname{sing}(M)$ is 0 . On other hand, the complexification of $\eta=i(\partial F-\bar{\partial} F)$ is

$$
\eta_{\mathbb{C}}=i\left(\partial_{z} F_{\mathbb{C}}-\partial_{w} F_{\mathbb{C}}\right) .
$$

Recall that $\left.\eta\right|_{M^{*}}$ and $\left.\eta_{\mathbb{C}}\right|_{M_{\mathbb{C}}^{*}}$ define $\mathcal{L}$ and $\mathcal{L}_{\mathbb{C}}$. Now we compute $\operatorname{sing}\left(\left.\eta_{\mathbb{C}}\right|_{M_{\mathbb{C}}^{*}}\right)$. We can write $d F_{\mathbb{C}}=\alpha+\beta$, with

$$
\alpha=\sum_{j=1}^{n} \frac{\partial F_{\mathbb{C}}}{\partial z_{j}} d z_{j}:=\frac{1}{2} \sum_{j=1}^{n}\left(\frac{\partial P}{\partial z_{j}}(z)+A_{j}\right) d z_{j}
$$

and

$$
\beta=\sum_{j=1}^{n} \frac{\partial F_{\mathbb{C}}}{\partial w_{j}} d w_{j}:=\frac{1}{2} \sum_{j=1}^{n}\left(\frac{\partial P}{\partial w_{j}}(w)+B_{j}\right) d w_{j},
$$

where $\frac{1}{2} \sum_{j=1}^{n} A_{j} d z_{j}=\sum_{j=1}^{n} \frac{\partial H_{\mathbb{C}}}{\partial z_{j}} d z_{j}$ and $\frac{1}{2} \sum_{j=1}^{n} B_{j} d w_{j}=\sum_{j=1}^{n} \frac{\partial H_{\mathbb{C}}}{\partial w_{j}} d w_{j}$.

Then $\eta_{\mathbb{C}}=i(\alpha-\beta)$, and so

$$
\left.\eta_{\mathbb{C}}\right|_{M_{\mathbb{C}}^{*}}=\left.\left(\eta_{\mathbb{C}}+i d F_{\mathbb{C}}\right)\right|_{M_{\mathbb{C}}^{*}}=\left.2 i \alpha\right|_{M_{\mathbb{C}}^{*}}=-\left.2 i \beta\right|_{M_{\mathbb{C}}^{*}}
$$

In particular, $\left.\alpha\right|_{M_{\mathbb{C}}^{*}}$ and $\left.\beta\right|_{M_{\mathbb{C}}^{*}}$ define $\mathcal{L}_{\mathbb{C}}$. Therefore $\operatorname{sing}\left(\left.\eta_{\mathbb{C}}\right|_{M_{\mathbb{C}}^{*}}\right)$ can be splited in two parts. Let $M_{1}=\left\{(z, w) \in M_{\mathbb{C}} \mid \frac{\partial F_{\mathbb{C}}}{\partial w_{j}} \neq 0\right.$ for some $\left.j=1, \ldots, n\right\}$ and $M_{2}=\left\{(z, w) \in M_{\mathbb{C}} \mid \frac{\partial F_{\mathbb{C}}}{\partial z_{j}} \neq 0\right.$ for some $\left.j=1, \ldots, n\right\}$, note that $M_{\mathbb{C}}=$ $M_{1} \cup M_{2}$; if we denote by

$$
X_{1}:=M_{1} \cap\left\{\frac{\partial P}{\partial z_{1}}(z)+A_{1}=\ldots=\frac{\partial P}{\partial z_{n}}(z)+A_{n}=0\right\}
$$

and

$$
X_{2}:=M_{2} \cap\left\{\frac{\partial P}{\partial w_{1}}(w)+B_{1}=\ldots=\frac{\partial P}{\partial w_{n}}(w)+B_{n}=0\right\},
$$

then $\operatorname{sing}\left(\left.\eta_{\mathbb{C}}\right|_{M_{\mathbb{C}}^{*}}\right)=X_{1} \cup X_{2}$. Since $P \in \mathbb{C}\left[z_{1}, \ldots, z_{n}\right]$ has an isolated singularity at $0 \in \mathbb{C}^{n}$, we conclude that $\operatorname{cod}_{M_{\mathbb{C}}^{*}} \operatorname{sing}\left(\left.\eta_{\mathbb{C}}\right|_{M_{\mathbb{C}}^{*}}\right)=n$.

If $n \geq 3$, we can directly apply Theorem 2.6 and the proof ends. In the case $n=2$, we are going to prove that $\mathcal{L}_{\mathbb{C}}$ has a non-constant holomorphic first integral.

We begin by a blow-up at $0 \in \mathbb{C}^{4}$. Let $F(x, y)=\mathcal{R} e(P(x, y))+$ h.o.t and $M=F^{-1}(0)$ Levi-flat. Its complexification can be written as

$$
F_{\mathbb{C}}(x, y, z, w)=\frac{1}{2} P(x, y)+\frac{1}{2} P(z, w)+H_{\mathbb{C}}(x, y, z, w) .
$$

We take the exceptional divisor $D=\mathbb{P}^{3}$ of the blow-up $\pi:\left(\tilde{\mathbb{C}}^{4}, \mathbb{P}^{3}\right) \rightarrow$ $\left(\mathbb{C}^{4}, 0\right)$ with homogeneous coordinates $[a: b: c: d],(a, b, c, d) \in \mathbb{C}^{4} \backslash\{0\}$. The intersection of the strict transform $\tilde{M}_{\mathbb{C}}$ of $M_{\mathbb{C}}$ by $\pi$ with the divisor $D=\mathbb{P}^{3}$ is the surface

$$
Q=\left\{[a: b: c: d] \in \mathbb{P}^{3} / P(a, b)+P(c, d)=0\right\},
$$

which is an irreducible smooth surface.

Consider for instance the chart $(W,(t, u, z, v))$ of $\tilde{\mathbb{C}}^{4}$ where

$$
\pi(t, u, z, v)=(t . z, u . z, z, v . z)=(x, y, z, w) .
$$


We have

$$
F_{\mathbb{C}} \circ \pi(t, u, z, v)=z^{k}\left(\frac{1}{2} P(t, u)+\frac{1}{2} P(1, v)+z H_{1}(t, u, z, v)\right),
$$

where $H_{1}(t, u, z, v)=H(t z, u z, z, v z) / z^{k+1}$, which implies that

$$
\tilde{M}_{\mathbb{C}} \cap W=\left(\frac{1}{2} P(t, u)+\frac{1}{2} P(1, v)+z H_{1}(t, u, z, v)=0\right)
$$

and so $Q \cap W=(z=P(t, u)+P(1, v)=0)$.

On the other hand, as we have seen in (3.2), the foliation $\mathcal{L}_{\mathbb{C}}$ is defined by $\left.\alpha\right|_{M_{\mathbb{C}}^{*}}=0$, where

$$
\alpha=\frac{1}{2} \frac{\partial P}{\partial x} d x+\frac{1}{2} \frac{\partial P}{\partial y} d y+\frac{\partial H_{\mathbb{C}}}{\partial x} d x+\frac{\partial H_{\mathbb{C}}}{\partial y} d y .
$$

In particular, we get

$$
\pi^{*}(\alpha)=z^{k-1}\left(\frac{1}{2} \frac{\partial P}{\partial x}(t, u) z d t+\frac{1}{2} \frac{\partial P}{\partial y}(t, u) z d u+\frac{1}{2} k P(t, u) d z+z \theta\right),
$$

where $\theta=\pi^{*}\left(\frac{\partial H_{\mathbb{C}}}{\partial x} d x+\frac{\partial H_{\mathbb{C}}}{\partial y} d y\right) / z^{k}$.

Hence, $\tilde{\mathcal{L}}_{\mathbb{C}}$ is defined by

$$
\alpha_{1}=\frac{1}{2} \frac{\partial P}{\partial x}(t, u) z d t+\frac{1}{2} \frac{\partial P}{\partial y}(t, u) z d u+\frac{1}{2} k P(t, u) d z+z \theta .
$$

Since $Q \cap W=(z=P(t, u)+P(1, v)=0)$, we see that $Q$ is $\tilde{\mathcal{L}}_{\mathbb{C}}$-invariant. In particular, $S:=Q \backslash \operatorname{sing}\left(\tilde{\mathcal{L}}_{\mathbb{C}}\right)$ is a leaf of $\tilde{\mathcal{L}}_{\mathbb{C}}$. Fix $p_{0} \in S$ and a transverse section $\sum$ through $p_{0}$. Let $G \subset \operatorname{Diff}\left(\sum, p_{0}\right)$ be the holonomy group of the leaf $S$ of $\tilde{\mathcal{L}}_{\mathbb{C}}$. Since $\operatorname{dim}\left(\sum\right)=1$, we can think that $G \subset \operatorname{Diff}(\mathbb{C}, 0)$. Let us prove that $G$ is finite and linearizable.

At this part we use that the leaves of $\tilde{\mathcal{L}}_{\mathbb{C}}$ are closed (see lemma 2.5).

Let $G^{\prime}=\left\{f^{\prime}(0) / f \in G\right\}$ and consider the homomorphism $\phi: G \rightarrow G^{\prime}$ defined by $\phi(f)=f^{\prime}(0)$. We assert that $\phi$ is injective. In fact, assume that $\phi(f)=1$ and by contradiction that $f \neq i d$. In this case $f(z)=$ $z+a . z^{r+1}+\ldots$, where $a \neq 0$. According to $[\mathrm{L}]$, the pseudo-orbits of this transformation accumulate at $0 \in\left(\sum, 0\right)$, contradicting that the leaves of $\tilde{\mathcal{L}}_{\mathbb{C}}$ are closed. Now, it suffices to prove that any element $g \in G$ has finite order (cf. [M-M]). In fact, if $\phi(g)=g^{\prime}(0)$ is a root of unity then $g$ has finite order because $\phi$ is injective. On the other hand, if $g^{\prime}(0)$ was not a root of unity then $g$ would have pseudo-orbits accumulating at $0 \in\left(\sum, 0\right)$ (cf. [L]). Hence, all transformations of $G$ have finite order and $G$ is linearizable.

This implies that there is a coordinate system $w$ on $\left(\sum, 0\right)$ such that $G=\langle w \rightarrow \lambda w\rangle$, where $\lambda$ is a $d^{t h}$-primitive root of unity (cf. [M-M]). In particular, $\psi(w)=w^{d}$ is a first integral of $G$, that is $\psi \circ g=\psi$ for any $g \in G$.

Let $Z$ be the union of the separatrices of $\mathcal{L}_{\mathbb{C}}$ through $0 \in \mathbb{C}^{4}$ and $\tilde{Z}$ be its strict transform under $\pi$. The first integral $\psi$ can be extended to a first integral $\varphi: \tilde{M}_{\mathbb{C}} \backslash \tilde{Z} \rightarrow \mathbb{C}$ be setting

$$
\varphi(p)=\psi\left(\tilde{L}_{p} \cap \sum\right)
$$

where $\tilde{L}_{p}$ denotes the leaf of $\tilde{\mathcal{L}}_{\mathbb{C}}$ through $p$. Since $\psi$ is bounded (in a compact neighborhood of $0 \in \sum$ ), so is $\varphi$. It follows from Riemann extension theorem 
that $\varphi$ can be extended holomorphically to $\tilde{Z}$ with $\varphi(\tilde{Z})=0$. This provides the first integral and finishes the proof of theorem 1 .

\section{Quasihomogeneous polynomials}

In this section, we state some general facts about normal forms of quasihomogeneous polynomials.

Definition 4.1. The Newton support of germ $f=\sum a_{i j} x^{i} y^{j}$ is defined as $\operatorname{supp}(f)=\left\{(i, j): a_{i j} \neq 0\right\}$.

Definition 4.2. A holomorphic function $f:\left(\mathbb{C}^{n}, 0\right) \rightarrow(\mathbb{C}, 0)$ is said to be quasihomogeneous of degree $d$ with indices $\alpha_{1}, \ldots, \alpha_{n}$, if for any $\lambda \in \mathbb{C}$ and $\left(z_{1}, \ldots, z_{n}\right) \in \mathbb{C}^{n}$, we have

$$
f\left(\lambda^{\alpha_{1}} z_{1}, \ldots, \lambda^{\alpha_{n}} z_{n}\right)=\lambda^{d} f\left(z_{1}, \ldots, z_{n}\right) .
$$

The index $\alpha_{s}$ is also called the weight of the variable $z_{s}$.

In the above situation, if $f=\sum a_{k} x^{k}, k=\left(k_{1}, \ldots, k_{n}\right), x^{k}=x_{1}^{k_{1}} \ldots x^{k_{n}}$, then $\operatorname{supp}(f) \subset \Gamma=\left\{k: a_{1} k_{1}+\ldots+a_{n} k_{n}=d\right\}$. The set $\Gamma$ is called the diagonal. Usually one takes $\alpha_{i} \in \mathbb{Q}$ and $d=1$.

One can define the quasihomogeneous filtration of the $\operatorname{ring} \mathcal{O}_{n}$. It consists of the decreasing family of ideals $\mathcal{A}_{d} \subset \mathcal{O}_{n}, \mathcal{A}_{d^{\prime}} \subset \mathcal{A}_{d}$ for $d<d^{\prime}$. Here $\mathcal{A}_{d}=\{Q:$ degrees of monomials from $\operatorname{supp}(Q)$ are $\operatorname{deg}(Q) \geq d\}$; (the degree is quasihomogeneous).

When $\alpha_{1}=\ldots=\alpha_{n}=1$, this filtration coincides with the usual filtration by the usual degree.

Definition 4.3. A function $f$ is called semiquasihomogeneous if $f=Q+F^{\prime}$, where $Q$ is quasihomogeneous of degree $d$ of finite multiplicity and $F^{\prime} \in \mathcal{A}_{d^{\prime}}$, $d^{\prime}>d$.

We will use the following result (cf. [A]).

Theorem 4.4. Let $f$ be a semiquasihomogeneous function, $f=Q+F^{\prime}$ with quasihomogeneous $Q$ of finite multiplicity. Then $f$ is right equivalent to the function $Q+\sum_{j} c_{j} e_{j}(z)$, where $e_{1}, \ldots, e_{s}$ are the elements of the monomial basis of the local algebra $A_{Q}$ such that $\operatorname{deg}\left(e_{j}\right)>d$ and $c_{j} \in \mathbb{C}$.

Example 4.5. If $f=Q+F^{\prime}$ is semiquasihomogeneous and $Q(x, y)=$ $x^{2} y+y^{k}$, then $f$ is right equivalent to $Q$. Indeed, the base of the local algebra $\mathcal{O}_{2} /\left(x y, x^{2}+k y^{k-1}\right)$ is $1, x, y, y^{2}, \ldots, y^{k-1}$ and lies below the diagonal $\Gamma$. Here $\mu(Q, 0)=k+1$.

\section{Proof of theorem 2}

Let $M=F^{-1}(0)$ be a germ at $0 \in \mathbb{C}^{n}, n \geq 3$ of real analytic Levi-flat hypersurface, where $F(z)=\mathcal{R} e(Q(z))+$ h.o.t and $Q$ is a quasihomogeneous polynomial of degree $d$ with an isolated singularity at $0 \in \mathbb{C}^{n}$. It is easily seen that $\operatorname{sing}\left(M_{\mathbb{C}}\right)=\{0\}$ and $\operatorname{cod}_{M_{\mathbb{C}}^{*} \sin g}\left(\mathcal{L}_{\mathbb{C}}\right) \geq 3$. The argument is essentially the same of the proof of theorem 1. In this way, there exists an unique germ at $0 \in \mathbb{C}^{n}$ of holomorphic codimension one foliation $\mathcal{F}_{M}$ tangent to $M$, moreover $\mathcal{F}_{M}: d h=0, h(z)=Q(z)+$ h.o.t and $M=(\mathcal{R} e(h)=0)$. Acoording 
to theorem 4.4, there exists $\phi \in \operatorname{Diff}\left(\mathbb{C}^{n}, 0\right)$ such that $h \circ \phi^{-1}(w)=Q(w)+$ $\sum_{k} c_{k} e_{k}(w)$, where $c_{k}$ and $e_{k}$ as above. Hence

$$
\phi(M)=\left(\mathcal{R} e\left(Q(w)+\sum_{k} c_{k} e_{k}(w)\right)=0\right) .
$$

\section{Applications}

Here we give some applications of theorem 1.

Example 6.1. $Q(x, y)=x^{2} y+y^{3}$ is a homogeneous polynomial of degree 3 with an isolated singularity at $0 \in \mathbb{C}^{2}$ and Milnor number $\mu(Q, 0)=4$. According to $[\mathrm{A}-\mathrm{G}-\mathrm{V}]$ pg. 184 , any germ $f(x, y)=x^{2} y+y^{3}+$ h.o.t is right equivalent to $x^{2} y+y^{3}$.

In particular, if $F(z)=\mathcal{R} e\left(x^{2} y+y^{3}\right)+$ h.o.t and $M=(F=0)$ is a germ of real analytic Levi-flat at $0 \in \mathbb{C}^{2}$, theorem 1 implies that there exists a holomorphic change of coordinate such that

$$
M=\left(\mathcal{R} e\left(x^{2} y+y^{3}\right)=0\right) .
$$

Example 6.2. If $Q(x, y)=x^{5}+y^{5}$ then $f(x, y)=Q(x, y)+$ h.o.t is right equivalent to $x^{5}+y^{5}+c . x^{3} y^{3}$, where $c \neq 0$ is a constant (cf. [A-G-V], pg. 194). Let $F(z)=\mathcal{R} e\left(x^{5}+y^{5}\right)+$ h.o.t be such that $M=(F=0)$ is Levi-flat, theorem 1 implies that there exists a holomorphic change of coordinate such that

$$
M=\left(\mathcal{R} e\left(x^{5}+y^{5}+c \cdot x^{3} y^{3}\right)=0\right) .
$$

\section{References}

[A] V.I. Arnold: "Normal Form of functions in the neighbourhood of degenerate critical points", UNM 29:2 (1974), 11-49, RMS 29:2 1948.

[A-G-V] V.I. Arnold, S.M. Gusein-Zade, A.N. Varchenko: "Singularities of Differential Maps", Vol. I, Monographs in Math., vol. 82, Birkhäuser, 1985.

[B-G] D. Burns, X. Gong: "Singular Levi-flat real analytic hypersurfaces", Amer. J. Math. 121, (1999), pp. 23-53.

[C-L] D. Cerveau, A. Lins Neto: "Local Levi-Flat hypersurfaces invariants by a codimension one holomorphic foliation". To appear in Amer. J. Math.

[L] F. Loray: "Pseudo-groupe d'une singularité de feuilletage holomorphe en dimension deux". Avaliable in http://hal.archives-ouvertures.fr/ccsd-00016434

[M-M] J.F. Mattei, R. Moussu: "Holonomie et intégrales premières", Ann. Ec. Norm. Sup. 13, (1980), pg. 469-523.

Instituto de Matemática Pura e Aplicada, IMPA

Current address: Estrada Dona Castorina, 110, 22460-320. Rio de Janeiro, RJ, Brazil.

E-mail address: afernan@impa.br 